F. Maloberti, M. Belloni, P. Malcovati: "Incremental Sigma-Delta Modulators for 3D-Imaging: System Architecture and Signal Processing"; 5th IEEE Conf. on Sensors, Daegu, October 2006, pp. 868-871.

(C)20xx IEEE. Personal use of this material is permitted. However, permission to reprint/republish this material for advertising or promotional purposes or for creating new collective works for resale or redistribution to servers or lists, or to reuse any copyrighted component of this work in other works must be obtained from the IEEE. 


\title{
Incremental Sigma-Delta Modulators for 3D-Imaging: System Architecture and Signal Processing
}

\author{
F. Maloberti, M. Belloni, P. Malcovati \\ Department of Electronics, University of Pavia, Pavia, Italy
}

\begin{abstract}
The design of a system for measuring the timeof-flight of a pulsed light beam for 3-D imaging with 2-4 ps resolution is described. An array of SPADs determines the arrival times of a single reflected photon measured by a coarse 10ns clock period and a fine of about 14 bit accuracy. The designed circuit uses an incremental second order $\Sigma \Delta$ modulator with a current and voltage feedback DAC on the integrators. The time-of-flight integrated circuit is for a $6.25 \mathrm{MHz}$ pulsed laser and a 2-D $32 \mathrm{x}$ 64 pixels SPAD array. The use of four time-to-digital converters enable obtaining 25 frame/s 3-D image with $23 \mathrm{~m}$ measurement range and $0.3 \mathrm{~mm}$ resolution.
\end{abstract}

\section{INTRODUCTION}

Many applications like face recognition, object and person monitoring, land and sea surveying, medical tomography imagers, requires using systems for capturing and rendering three-dimensional (3-D) images. Since stereoscopic systems require a non-negligible distance between the 2-D optical arrays and a significant signal processing, there is an increasing activity on non non-stereoscopic 3- $D$ vision systems that use a 2-D array of optical sensors and different approaches to obtain the third dimension. Among them, triangulation, interferometry, and pulsed time-of-flight $(T o F)$. Since triangulation and interferometer based methods are problematic and the cost is still prohibitive for many applications, $T o F$ is preferable [1].

The $T o F$, also used in laser range-finder [2], is applied to all the pixels of the 2-D array. It operates by transmitting a short laser pulse to an optical visible target and by detecting the reflected pulse by an optical detector. The measured distance is then calculated from this flight time. Fig. 1 shows the basic blocks and the principle of the pulsed laser $T o F$ which consists of a time interval measurement controller $(T D C)$ and receiver channel, laser and microcontroller for the laser driving and the 3-D image representation.

The simplest processing for a $T D C$ is just a counter of a given clock reference $f_{C K}$. The resolution of such a $T D C$ is limited by the period of the clock $T_{C K}$. If the timing signals are asynchronous to the measuring instants, the resolution can be improved by averaging several results from the same distance. The pulsed laser must operate with a frequency that is much higher than the band of the signal to obtain the required oversampling used by the averaging method.

This paper describes a $T D C$ system that better exploiting the oversampling obtains high spatial resolution by achieving

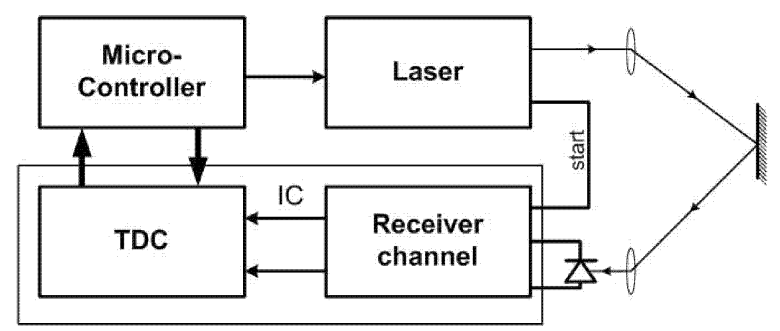

Fig. 1. Generic scheme of a pulsed laser TOF system.

the equivalent of an incremental $\Sigma \Delta$ modulator. The circuit designed using a $0.18 \mu \mathrm{m} C M O S$ technology is currently under fabrication. The features and expected performances are described in the following sections.

\section{SySTEM ARChiteCTURE}

The 3- $D$ system that this paper addresses is made by an array of 32 x 64 single-photon avalanche diodes $(S P A D)$ whose implementation on a single chip has been demonstrated using a high-voltage $0.8 \mu \mathrm{m} C M O S$ process with dual $\mathrm{p}+/$ deep n-tub, $\mathrm{n}$-tub/p-substrate junction. The upper $\mathrm{p}+/$ deep $\mathrm{n}$-tub junction obtains the multiplication region for the Geiger controlled breakdown. The process, that allows an operating voltage up to $50 \mathrm{~V}$, realizes a single $S P A D$ pixel using a $60 \times 60 \mu \mathrm{m}$ area. It is expected that a the new generation of the same technology, $0.35 \mu \mathrm{m} C M O S$, enables reducing the pixel area down to $30 \times 30 \mu \mathrm{m}$. Therefore, the area for the array and the required control logic is an affordable $2.5 \times 1.5 \mathrm{~mm}$ chip. The array operates and provides the output of only 4 pixel per clock period. Moreover, for each pixel the TDC needs 256 successive measures for obtaining the required accuracy. Therefore, for a complete frame $(32 \times 62) / 4 \times 256=131,072$ clock periods are necessary. In this way, a clock frequency of $6.25 \mathrm{MHz}$ enables about 50/frame per second.

The block diagram of the architecture is the three chips system of Fig. 2. The TDC processes the low jitter clock and generates the phases used by the $S P A D$ array and receives the signals of the 4 activated $S P A D s$. Every 256 clock periods the $T D C$ generates four 13 -bit plus time measures, sent to the $D S P$ system for generating the 3-D image displayed on the screen. 


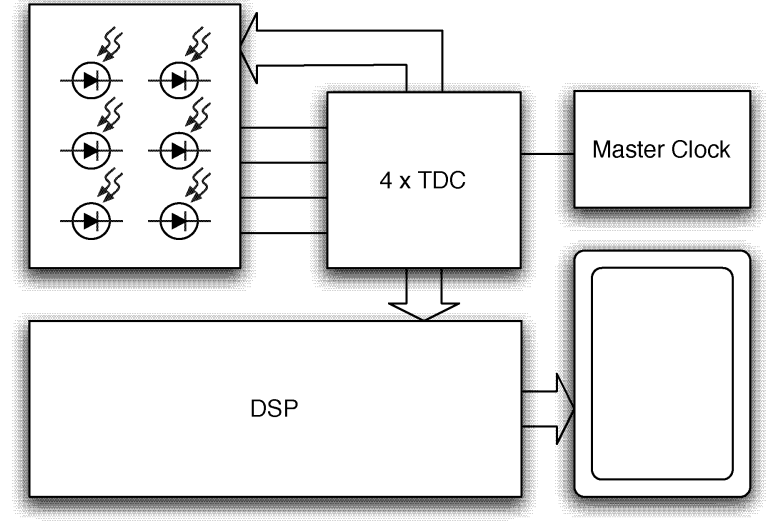

Fig. 2. Block diagram of the proposed 3-D architecture.

\section{TDC OPERATION}

The measure of the time is based on the master clock synchronous with the start pulses as shown in Fig. 3. The coarse measure is at the end of the clock period during which the stop pulse occurs. The stop pulse is also the beginning of the fine time measure $t_{f}$ that ends at the end of the period of the clock. Therefore, the measure time $t_{m}$ is

$$
t_{m}=k T-t_{f}
$$

Since the used master clock is $100 \mathrm{MHz}$ the coarse accuracy is $10 n s$. In order to have an accuracy of $2 p s$ it is necessary to obtain at least 12.3-bit accuracy. The spatial resolution with $2 \mathrm{ps}$ of accuracy is therefore $0.3 \mathrm{~mm}$. As we will see shortly the measure of the fine time is actually the measure of the area of the fine pulse. A possible finite rise and fall time makes trapezoidal the shape of the pulse that becomes a triangle for pulse duration smaller than the rise or the fall time. Since when the pulse becomes a triangle the area diminishes quadratically with its base, a non linear error occurs. In order to avoid this the system extends the duration of the fine pulse $t_{s}$ by $T$ doubling the full scale of the fine period and requiring an extra bit of accuracy. Therefore, the target of this design is to achieve 13.3-bit in the fine measurement. A possible difference between rise and fall time causes an offset error that is normally inessential in the target applications.

\section{Circuit Design}

The measure of the fine pulse is done with the following strategy:

- generation of a pulse of current with duration equal to the fine pulse;

- integration of the current to obtain an input charge;

- use of this charge as input of an initially reset 1-bit second order $\Sigma \Delta$ modulator;

- post-processing of a 256 bit-stream of digital data.

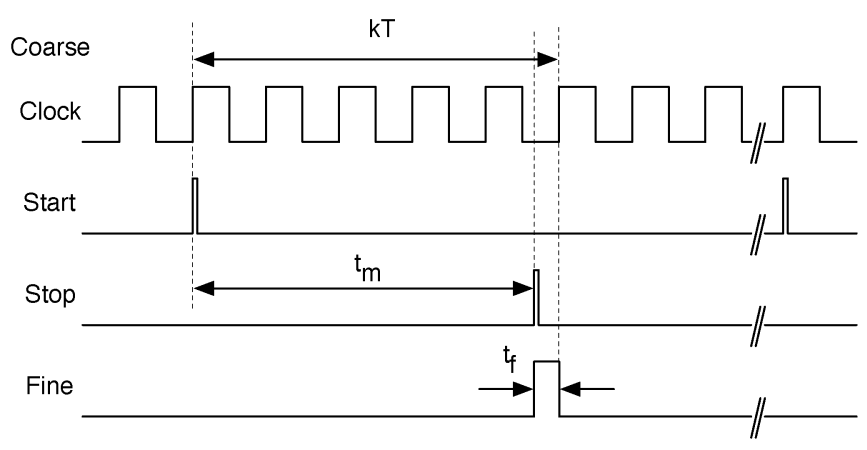

Fig. 3. Timing diagram of the TDC.

\section{A. Current Pulse Generators}

The system requires two current pulses one drained from $V_{D D}$ and controlled by the fine pulse; the other sinking current to ground with a duration equal to $2 T$ and controlled by the bit stream generated by the $\Sigma \Delta$ modulator. The scheme of Fig. 4 obtains the two current pulses. It uses two low-noise op-amp and low noise references to obtain clean currents that are switched toward the output or a dummy node by the four steering current devices controlled by the indicated phases. The two reference voltages are regulated at the start-up for making the drain current equal to the sink current.

The switching of the drain current source is similar to the one of the charge pump used in PLL and occurs for at least $10 \mathrm{~ns}$. The switching of the sink current occurs for $20 \mathrm{~ns}$. The used $0.18 \mu \mathrm{m}$ technology is adequate for the required switching speed.

\section{B. Integration of the Current}

Since the input stage of a $\Sigma \Delta$ modulator is an integrator it is not necessary to have a specific block for the integration of the current. It is just necessary to use the output of the current pulse generator as the input of the $\Sigma \Delta$ whose input stage operates in the current mode.

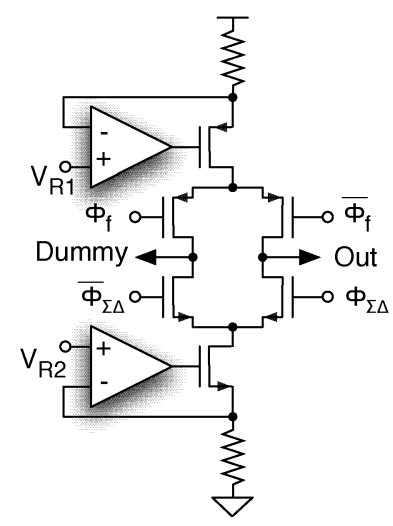

Fig. 4. Generator of current pulses 


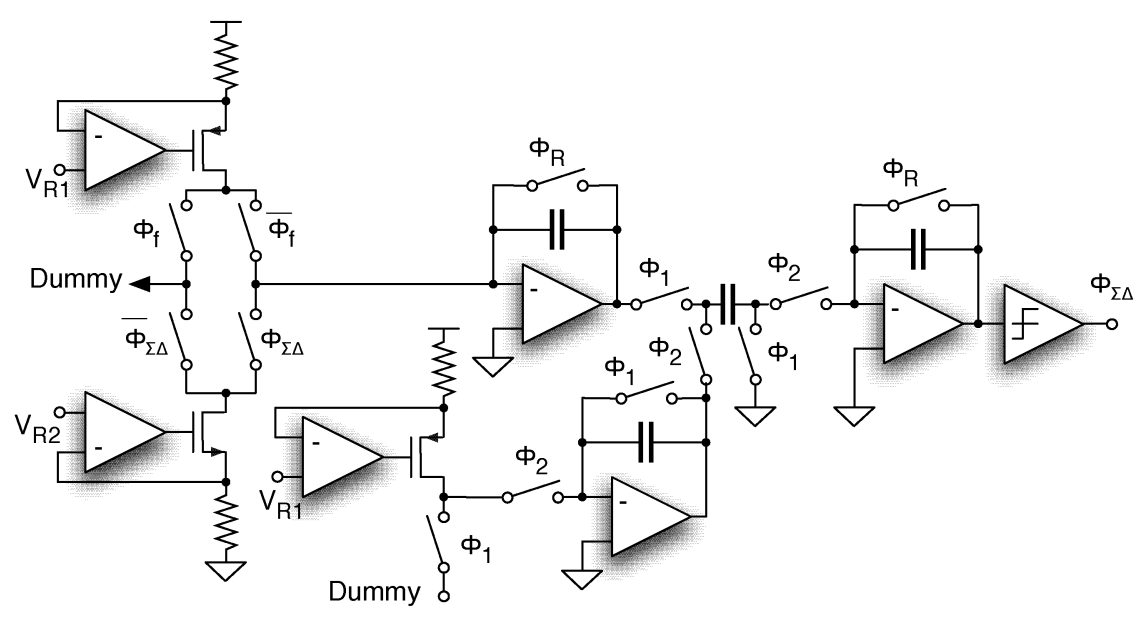

Fig. 5. Schematic of the entire TDC.

\section{Sigma-Delta Modulator}

Since the band of a signal of $3-D$ imagers is very slow with respect to the used $6.25 \mathrm{MHz}$ the input pulse for the foreseen 256 clock period can be assumed constant. Therefore, the quantization error of the 1-bit quantizer cannot be assumed equivalent to noise making difficult to exploit the noise shaping. The problem was extensively studied and a possible solution is using high order modulators and dither for breaking the limit cycles that occur at given input levels. The choice of this implementation is a second order modulator and no dither as the noise affecting the input signal caused by air turbulence and the SPAD is enough for our purposes.

The used scheme has a delayed switched capacitor integrator as second stage. A possible error in the reference voltage of the second stage has a limited impact on the transfer functions; however, in order to minimize that error the reference generator is obtained by a replica of the drain current source switched for $2 T$ into the virtual ground of an integrator matching the input stage of the $\Sigma \Delta$. A reset discharges the integrating capacitance every clock period. The integration of the current during the phase $\Phi_{2}$ obtains a ramp instead than a fixed voltage. The effect is the same as the one of a fixed voltage but the slew-rate requirements of the second op-amp are relaxed. The overall schematic is shown in Fig. 5.

\section{Post Processing}

A conventional incremental converter uses a first order modulator and a digital accumulator to obtain the digital output. In order to obtain $n$ bit of accuracy it is necessary to accumulate the output for $2^{n}$ clock periods. Therefore, for obtaining 14-bit it would be necessary using 16,384 clock periods. The approach used in this circuit is more effective as it exploits the obtained noise shaping in a way similar to the method proposed in [3].

By inspection of the circuit of Fig. 5 the output of the second integrator in the time domain is given by

$$
V_{2}(n)=\sum_{m=0}^{n} \sum_{k=0}^{m}\left[V_{\text {in }}(n)-V_{\text {out }}(n)\right]-\sum_{m=0}^{n} V_{\text {out }}(n)
$$

where $V_{\text {out }}(n)=V_{\text {ref }} \cdot \Phi_{\Sigma \Delta}$. Since the modulator is stable $V_{2}$ is limited and, according to simulation its value is, for any input in the interval $\pm 0.5 V_{F S},-2 V_{F S}<V_{2}<2 V_{F S}$.

If the input signal is constant over the measurement periods after $n$ clock periods the first term in the double summation give rise to $V_{i n} n \cdot(n+1) / 2$. Moreover if $n$ is large enough the simple accumulation of $V_{\text {out }}(n)$ is negligible. Therefore

$$
\frac{n \cdot(n+1)}{2} V_{\text {in }}=V_{2}(n)+\sum_{m=0}^{n} \sum_{k=0}^{m} V_{\text {out }}(n)
$$

The above result shows that the double summation (obtained with the cascade of two discrete time integrators - CoI) obtains the measure of $V_{i n}$ with an error lower than $2 \cdot \operatorname{Max}\left(V_{2}\right) /\{n$. $(n+1)\}$. Since the input must be in the interval $\pm 0.5 V_{F S}$, the minimum expected number of bits is $\log _{2}[n \cdot(n+1) / 4]$.

The use of $n=256$ obtains about 14-bit, not enough for our target. However, it is possible to increase the accuracy by a suitable additional processing. The method of [3] uses an extra integrator in the CoI. This is equivalent to the accumulation of terms given by (3) with $k$ instead of $n k=1 \cdots n$. The errors given by $V_{2}(k)=\epsilon_{V_{2}} \neq 0$ are also piled up. Since the errors can be positive or negative the total effect can be for fortunate input levels zero. We can assume that the accumulated value is within $\sqrt{n}$ times the input range, $\pm 0.5 V_{F S}$. For 256 samples the expected number of bits with a cascade of three integrators increases to 16.9-bit.

Observe that the first terms of the extra integrators are not very effective because each of them contributes with a small coefficient $k \cdot(k+1) / 2$ but causes a $\epsilon_{V_{2}}$ noise. Therefore, the benefit due to the extra processing is negligible at the beginning of the extra summation. This observation suggests to start the extra integration when the coefficient $k \cdot(k+1) / 2$ 


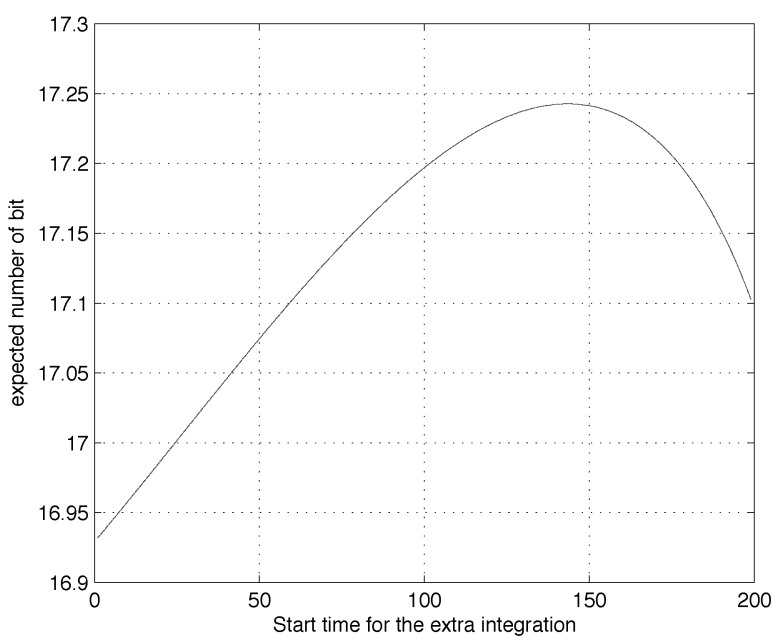

Fig. 6. Number of bits for reduced extra integration.

is large enough to determine a solid benefit. Fig. 6 shows the expected number of bits as a function of the starting point of the extra integrator. Observe that with the method proposed in [4] the expected resolution increases from 14-bit to 16.9-bit. However starting the third accumulation after about 150 clock periods out of 256 obtains a slightly better result (17.25 bit) and reduces the computation efforts.

The above considerations are approximate as the error caused by the non zero voltages at the second integrator output is not a random variable. Therefore, it is necessary to estimate the resolution of the measure by computer simulations.

\section{Simulation RESUlts}

The TDC converter has been extensively simulated at the behavioral and the transistor level. The used technology is a $0.18 \mu \mathrm{m} \mathrm{CMOS}$ with 1 -poly and 5 metals.

The first design concern is to have a very high accuracy in the charge pump for avoiding degradation of performances. Simulation shows that the distortion caused by the finite output

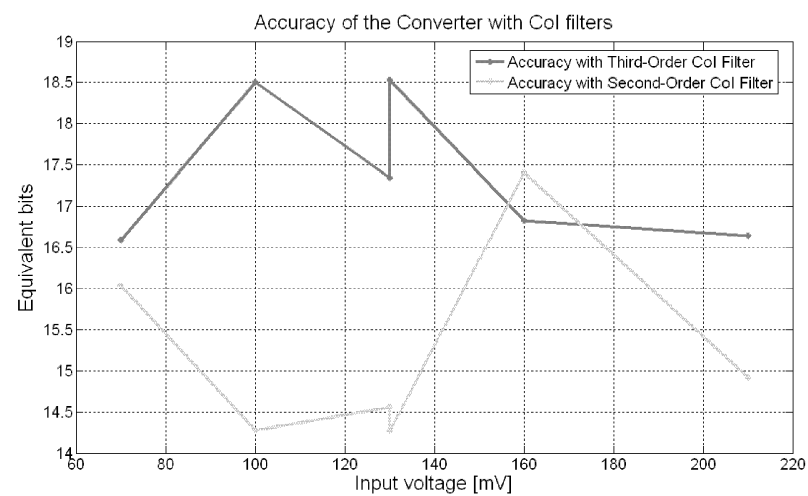

Fig. 7. Equivalent number of bit of the TDC as a function of the input voltage (or time delay)

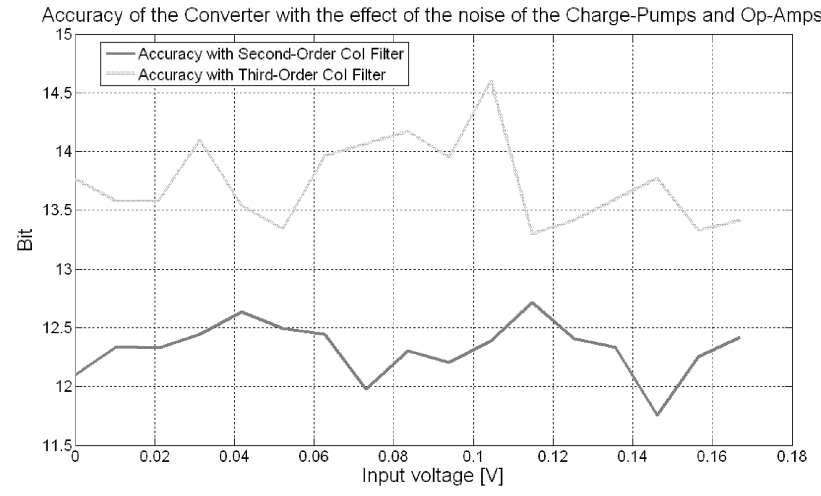

Fig. 8. Equivalent number of bit of the TDC as a function of the input voltage (or time delay) with electronic noise.

resistance and real op-amp and switches is few ppm making the designed charge pump well suitable for a 13 -14-bit target.

The simulations of the entire scheme at the transistor level that include the limitations of the finite gain, bandwidth and slew-rate of the op-amps give rise to the result of Fig. 7. The use of a CoI with 2 integrators gives rise to about 14-15 bit (the predicted value). The use of a third integrator increases the resolution to about $17-18$ bit that is again in line with the approximated analysis done in the previous section.

The obtained resolution does not account for the thermal noise of the active and passive devices and for the $k T / C$ noise. The simulation at the transistor level with all the noise sources included diminishes the number of bits to 12 bit and 13.5 bit for the two $\mathrm{CoI}$ and $3 \mathrm{CoI}$ respectively. Therefore, since the full scale of the $T D C$ is $20 \mathrm{~ns}$, the expected resolution with 3 CoI is 2 ps.

\section{CONCLUSIONS}

A system for measuring the time of flight in a 3-D system that obtains $2 p s$ resolution is described. The system has been studied at the architecture level and the key block the TDC at the transistor level. The circuit is being integrated and silicon is expected by the end of the year. The chip active area is 1.1 $x 1.6 \mathrm{~mm}$, the power consumption is $5.8 \mathrm{~mW}$ at $1.8 \mathrm{~V}$ supply voltage.

\section{REFERENCES}

[1] C. Niclass, A. Rochas, P. Besse, and E. Charbon, Design and characterization of a CMOS 3-D image sensor based on single photon avalanche diodes, IEEE Journal of Solid-State Circuits, vol. 40, pp. $1847-1854,2005$.

[2] J. Nissinen, J. Kostamovaara, Wide dynamic range CMOS receivers for a pulsed time-of-flight laser range finder, Instrumentation and Measurement Technology Conference, Vol. 2, pp. 1224 - 1227, 2004.

[3] J Markus, J. Silva, G. C. Temes, Theory and Applications of Incremental Converters, IEEE Trans. on Circuit and Systems - I: Vol. 51, pp. $678-690,2004$.

[4] V. Quiquempoix, P. Deval, A. Barreto, G. Bellini, G. J. Markus, J. Silva,G. C. Temes, A low-power 22-bit incremental ADC, IEEE Journal of Solid-State Circuits, vol. 41, pp. 1562 - 157, 2006. 